\title{
Délibérations du Comité central de la FMH
}

St. Lors de sa séance du 16 décembre 1999, le Comité central (CC) a traité, entre autres, des affaires suivantes:

\section{Assurances sociales}

1.1 Dénonciation formelle de la convention d'introduction du TarMed

Le but fixé par la convention sur l'introduction du TarMed, signée le 16 avril 1999, sera atteint au début de l'an 2000 par la publication de la structure tarifaire TarMed alpha 2.2. Le moment sera alors venu pour la nouvelle organisation TarMed de prendre le relais pour assurer le développement futur et le contrôle de la structure tarifaire. De plus, le budget prévu pour l'introduction du TarMed étant pratiquement épuisé, la FMH, qui doit s'en tenir aux dispositions budgétaires, ne peut apporter son soutien financier qu'à une nouvelle organisation TarMed. Le CC décide donc d'intervenir auprès des partenaires tarifaires pour que la convention concernant cette nouvelle organisation soit signée dans les plus brefs délais, créant ainsi les conditions permettant de dénoncer la convention actuelle.

\section{Assurance qualité}

2.1 Consultation sur le Guide de pratique médicale concernant le carcinome mammaire

Le groupe de travail interdisciplinaire "diagnostic" a élaboré, se fondant en cela sur le modèle canadien, un guide de pratique médicale concernant le diagnostic et le traitement du carcinome mammaire. Celui-ci a été mis en consultation auprès des sociétés de discipline médicale concernées qui sont priées de donner leur appréciation d'ici fin 1999. Cette consultation, au sens d'un processus consensuel et d'un réexamen, sera suivie d'une phase clinique test. La publication du guide n'interviendra qu'au terme de ces étapes.

\section{Formation postgraduée et continue}

3.1 Adjonction à la convention avec Swisscom sur les mentions autorisées dans l'annuaire téléphonique sous la rubrique "Médecins"

Le CC décide de compléter la convention avec les mentions suivantes:

- Gériatrie

- Médecine d'orientation anthroposophique

- Electroencéphalographie (EEG)
- Electroneuromyographie

- Hypnose médicale

- Médecine psychosomatique et psychosociale

- Thérapie neurale

Le CC prend la décision simultanément de n'admettre aucune mention concernant les certificats d'aptitude technique, notamment dans le domaine de l'ultrasonographie, se conformant ainsi à l'exigence de la Chambre médicale de faire preuve de retenue dans la création de nouvelles mentions.

\subsection{Examen de spécialiste obligatoire}

Le CC ratifie la mise en vigueur au $1^{\text {er }}$ janvier 2000 de l'obligation de réussir l'examen de spécialiste obligatoire pour la médecine générale et la gastroentérologie.

3.3 Commission d'experts pour l'examen intercantonal de chiropraxie

Le CC relève que la FMH est seule compétente pour la désignation de ses délégués dans les diverses commissions où elle est représentée. Cela étant, il se réserve donc le droit de mandater un médecin de son choix comme expert aux examens des chiropraticiens.

\subsection{Programmes de formation continue}

L'homogénéité des programmes, la qualité des sessions de formation continue, le montant des crédits et ce qu'on appelle le tourisme de formation continue sont autant de sujets qui font l'objet de discussions soutenues au sein du CC. Il se prononce donc pour l'élaboration d'un concept général, prévoyant notamment l'évaluation unifiée des sessions de formation continue, la promotion de méthodes audiovisuelles dans les programmes de formation continue, des systèmes de contrôles, l'homogénéité des programmes ainsi que des ébauches de solution aux problèmes encore à régler.

Le CC adopte en outre les programmes de formation continue suivants:

- Allergologie et immunologie clinique (sous réserve de retouches rédactionnelles)

- Chirurgie de la main (sous réserve de retouches rédactionnelles)

- Infectiologie

- Pharmacologie clinique (sous réserve de retouches rédactionnelles)

- Oncologie-hématologie pédiatrique (suppression des deux derniers points du chiffre 4)

- Radiologie pédiatrique (suppression de la $2^{\mathrm{e}}$ partie du chiffre 12)

Toutes les sociétés de discipline médicale disposent maintenant d'un programme de formation continue agréé par le CC.

\subsection{Examen écrit 1999 de la SSMI}

L'examen écrit 1999 de la SSMI a donné lieu à des critiques. Le CC a chargé le groupe d'experts FAP d'examiner le bien-fondé de ces critiques et de se prononcer à cet égard. 


\section{Organisation}

4.1 Nouvelles pratiques des tribunaux genevois Il semble que, de plus en plus fréquemment, des médecins ayant fonctionné comme expert désigné par le Bureau d'expertises extrajudiciaires de la FMH sont invités après coup par les tribunaux genevois à répondre en qualité de témoin à un catalogue de questions détaillé. Le CC a eu connaissance que les tribunaux genevois ont eu et ont toujours recours à de telles pratiques à propos du canton du Valais. Il constate qu'il s'agit d'un usage abusif de l'expert en tant que témoin et que ces pratiques, jusqu'ici propres au canton de Genève seulement, peuvent constituer une menace pour le Bureau d'expertises extrajudiciaires de la FMH.

Le CC fera part de ces considérations aux départements cantonaux de justice et un article sur ce thème fera l'objet d'une publication dans le BMS.

\subsection{Médicaments coûteux}

Une tendance se dessine visant à mettre sur le marché dans les années à venir un nombre toujours plus grand de médicaments coûteux, destinés à de petits groupes de patients bien précis. Jusqu'ici ces médicaments étaient prescrits presque exclusivement par des médecins très spécialisés. Il faut savoir cependant qu'en vertu de la liberté de traitement que l'on connaît en Suisse, tout médecin est autorisé à prescrire de tels médicaments. Les cercles de spécialistes ne sont toutefois pas univoques sur l'importance et notamment les dangers des prescriptions à long terme. Selon eux, les indications devraient être très restreintes et posées uniquement par des spécialistes hautement qualifiés. A cet égard, citons l'exemple du Danemark qui tient un registre facultatif de tous les patients recevant un médicament particulier pour un traitement défini. En Suisse également, certains milieux se disent préoccupés par la possibilité qu'ont les médecins de prescrire ces médicaments coûteux.

Le CC laisse le soin aux sociétés de discipline médicale de décider si elles entendent faire des propositions à la Conférence des présidents et, partant, à la Chambre médicale à ce sujet.

4.3 Révision de la loi sur les stupéfiants Le CC adopte la prise de position de la FMH à l'intention de la cheffe du Département fédéral de l'Intérieur (DFI) dans laquelle il a été tenu compte des remarques des sociétés cantonales de médecine et des sociétés de discipline médicale.

\subsection{Emission "10 vor 10» (DRS) du 24 novembre} 1999

Le président et le secrétaire général de la FMH ont écrit à M. Marc André Giger, directeur du Concordat des assureurs maladie suisses (CAMS) pour le prier de bien vouloir prendre position sur les propos qu'il a tenu lors de l'émission "10 vor 10" du 24 novembre 1999 selon lesquels il n'existe aucune autre profession garantissant un revenu de 200000 .- francs à la sortie des études.

\section{Weiterbildungsstätten / Arztpraxen}

\section{Korrektur / Ergänzung zur publizierten Liste in Nr. 1/2000}

Bezüglich der neuen Arztpraxen ist folgende Korrektur bzw. Ergänzung zu machen:

\section{Allgemeinmedizin}

Samnaun, Dr. med. Rudolph Horn

\author{
Allgemeinmedizin, chirurgische/ orthopädische \\ Praxen \\ (gilt nicht für chirurgisches Pflichtjahr) \\ Winterthur, Dr. med. Luzi Dubs \\ Kann für den Facharzttitel FMH für Allgemeinmedi- \\ zin nur für das 2. Wahljahr angerechnet werden!
}

